\title{
Nouvelles données biogéographiques sur Bombus jonellus (KIRBY 1802) (Hymenoptera, Apidae) dans les Pyrénées
}

\author{
Par David GENOUD * et Pierre RASMONT **
}

\begin{abstract}
New biogeographical data on Bombus jonellus (KIRBY 1802) (Hymenoptera: Apidae) in the Pyrenees. Bombus jonellus is very rare in the Pyrenees. So far, it is known only in the western part of these mountain range. We here provide several new locations and give indications about the habitat preferences and foraging plants of this bumblebee species. Some suggestions to promote the conservation of these isolated populations are made.
\end{abstract}

Résumé. Bombus jonellus est très rare dans les Pyrénées. Il n'est connu à ce jour que de la partie occidentale de cette chaîne montagneuse. Nous fournissons ici plusieurs nouvelles localités et des indications à propos des préférences d'habitat et de fleurs butinées par cette espèce de bourdon. Nous donnons aussi quelques suggestions pour la conservation de ces populations isolées.

Mots-clés. Bombus jonellus, Apidae, Pyrénées.

\section{Introduction}

Bombus jonellus est un petit bourdon à très vaste distribution mais plutôt nordique (Løken 1973). Il abonde dans la taïga depuis la Norvège jusqu'au Kamtschatka et même jusqu'en Alaska et au Canada (Williams 1998). Il se trouve aussi dans les landes atlantiques de l'Europe Occidentale, surtout dans les îles Britanniques (Alford 1975). Plus au sud, il devient franchement montagnard comme dans les Alpes et le Massif Central (Delmas 1976; Amiet 1996). Il est connu d'Espagne aux Monts Cantabriques (Løken 1973; Reinig 1976; Ornosa 1984) et des Pyrénées (Ornosa 1984; Rasmont 1986). C'est une des rares espèces de bourdons bivoltines (Løken 1973). Il butine les myrtilles (Vaccinum myrtillus), les airelles ( $V$. vitis-idaea), les canneberges ( $V$. uliginosus), d'autres Ericaceae et, occasionnellement les ronces (Rubus spp.).

Sa distribution en France est présentée par Rasmont et al. (2002) et par Mahé \& Rasmont (2008). Il est resté longtemps ignoré des Pyrénées Occidentales où ce n'est qu'en 1983 (Rasmont 1986) qu'il a été découvert (Hautes-Pyrénées, Aucun, Forêt d'Arragnat). L'espèce était auparavant inconnue d'Aquitaine (Pérez 1890, 1905).

A l'occasion de ses premières observations dans la région, Rasmont (1986) a synthétisé les informations biogéographiques, écologiques et systématiques concernant cette espèce et décrit la ssp. yarrowianus (Rasmont 1986) pour inclure les populations des Monts Cantabriques et des Pyrénées.

* David Genoud, 432 Route des Pyrénées, F-40390 Saint-Laurent-deGosse, dge-davidgenoud@orange.fr

** Pierre Rasmont, Laboratoire de Zoologie, Université de Mons,

Place du Parc 20, B-7000 Mons, Belgique, pierre.rasmont@umh.ac.be
Lors de nos discussions nous avions évoqué la nécessité de rechercher cette espèce dans ces milieux favorables aquitaniens en plaine comme en montagne. Nous avons longuement recherché l'espèce en plaine (PR en 1993, DG en 2005-2007). Des collections d'entomologistes locaux ont été consultées, sans succès. $\mathrm{Ni}$ les landes atlantiques de plaines, à éricacées mais sans Vaccinum (départements des Landes et de la Gironde), ni la forêt de la Double (Dordogne) ne nous ont semblé accueillir l'espèce.

Fin juillet 2007, dans le cadre d'une collaboration à un inventaire en forêt d'Iraty, DG et NJ Vereecken ont visité le versant français de ce massif forestier sur les communes de Lécumbery, Esterençuby, Mendive et Larrau (Pyrénées-Atlantiques). Les habitats y semblent favorables avec une mosaïque de landes à éricacées et de sous bois à Vaccinum spp.. Là encore, l'espèce n'est pas détectée.

Le 10 septembre 2007, DG est retourné prospecter ce massif à la recherche des mâles de différents bourdons susceptibles d'être présents à cette époque. C'est alors que quelques individus de Bombus jonellus ont été contactés et capturés sur la commune de Larrau (Crête d'Orgambidesca, 1 mâle sur Erica cinerea) (Figure 3) et de Mendive, (tourbière de Surzay, 1 ouvrière sur Erica cinerea et col d'Oraaté, 1 ouvrière sur Calluna vulgaris).

Sur base d'une recherche bibliographique sur les stations Navarraises de l'espèce (Burguete) (Ornosa 1984), l'un de nous (DG) est allé les 16 et 17 septembre 2007 faire une visite plus approfondie du massif montagneux entre la vallée des Aldudes et le Massif d'Iraty. Ainsi le 16 septembre l'espèce est découverte en abondance à la frontière franco-espagnole sur la 


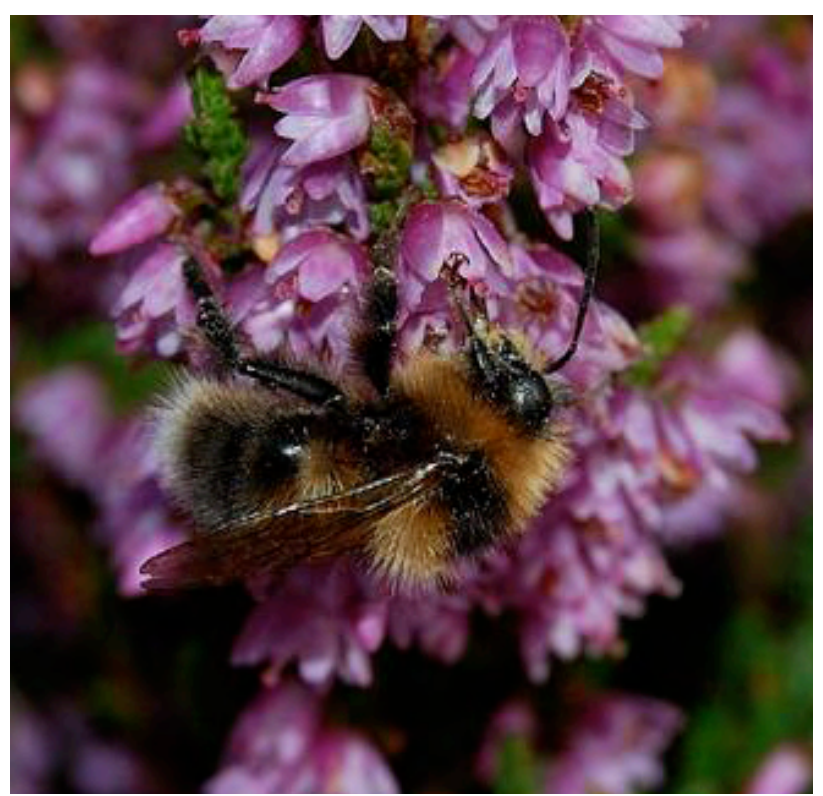

Figure 1. Bombus jonellus mâle, 16/09/2007, Espagne, Burguete, Redoute de Trona. (Photo David Genoud)

Redoute de Trona (Burguete, 1200 m.). Plus d'une cinquantaine de mâle mais aussi quelques ouvrières sont observées. Là l'espèce s'affaire principalement sur Calluna vulgaris. Cette station est située à quelques kilomètres de celle de Mezkiriz (commune de Burguete) lieu de découverte de l'espèce sur le versant espagnol du massif pyrénéen (Rasmont 1986). Le versant Espagnol de la Redoute de Lindux permet de collecter un autre mâle ainsi que le Col de Burdincurutcheta (est), côté français (commune des Aldudes). Les landes de la forêt d'Hayra (Aldudes) à $800 \mathrm{~m}$ d'altitude sont également prospectées mais sans succès, et ce malgré l'abondance d'autres espèces des bourdons sur les éricacées. Dans le même secteur, plus au Nord, les landes frontalières de la crête d'Achistoy sont visitées et permettent là encore quelques observations et captures.

L'espèce est observée ainsi côté espagnol jusqu'au col d'Ibaneta (Puerto de Ibaneta) qu'il n'atteint pas. La montagne d'Ortzanzurrieta n'a pas été visitée.

Sur la haute route qui mène vers la partie française de la forêt d'Iraty, ce n'est qu'à partir de la forêt d'Orion que les habitats redeviennent favorables sur le versant Français sans que l'espèce n'y ait été contactée. Toutefois la visite effectuée en fin de journée peut avoir été quelque peu tronquée.

Le 17 septembre 2007 est consacré au contrôle de stations découvertes le 10 septembre et a quelques extensions de la prospection sur le massif. Ainsi l'espèce est retrouvée en abondance sur la crête d'Orgambidesca à Pellusegagna, au col d'Oraaté. L'espèce est absente des landes de la crête de Milagatte et de Tharta. On trouve Bombus magnus sur ces landes plus isolées de la partie boisée du massif.

Toutes les données connues de Bombus jonellus pour les Pyrénées sont reprises dans le Tableau 1. La carte

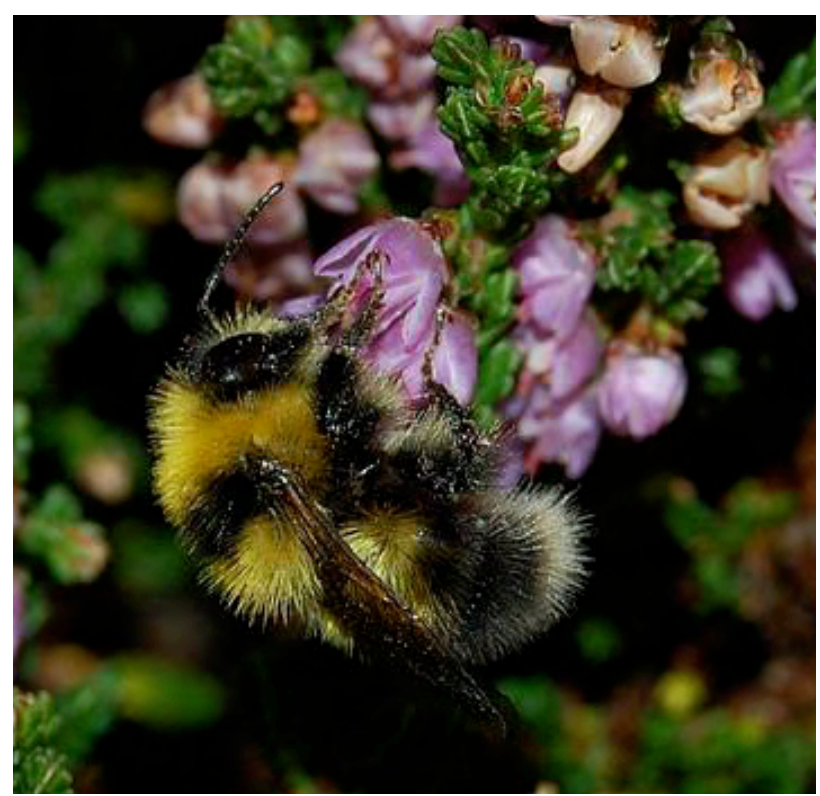

Figure 2. Bombus jonellus mâle, 10/09/2007, France, Lécumberry, Col d'Oraaté. (Photo David Genoud)

de distribution est présentée à la Figure 6. Tout indique qu'il s'agit bien partout de la ssp. yarrowianus.

\section{Discussion}

La découverte dans une succession de vallées et de massifs forestiers d'un peuplement prospère de Bombus jonellus laisse entrevoir une répartition plus large encore sur la crête occidentale des Pyrénées.

On sait maintenant que $B$. jonellus est présent dans toute une série de stations des Pyrénées Occidentales. Il y reste cependant plutôt rare, localisé et restreint à des biotopes bien typés (toujours riches en Ericaceae). Malgré les recherches intenses, on ne l'a pas trouvé dans le Parc National des Pyrénées (Iserbyt sous presse), nulle part dans la partie orientale de la chaîne des Pyrénées, comme à Nohèdes (Gosselin et al. 2007), à Eyne (Iserbyt et al. 2008), à la Massane (Rasmont 1999), ni nulle part dans les Pyrénées catalanes (Rasmont 1988).

Il n'est pas exclu que le petit nombre d'observations de cette espèce soit le résultat de sa phénologie bivoltine. En effet, la plupart des données sont soit précoces (mai-juin) soit tardive (septembre), tandis que le coeur de l'été (juillet-août) ne permet pas de l'observer en nombre.

Quoi qu'il en soit, l'espèce est rare dans les Pyrénées.

La reprise des données anciennes de Navarre nous montre par ailleurs que les données de Trona et Mezkiritz (Burguete) sont très proches de la vallée des Aldudes. La station de Mezkiritz (en basque ou Mezquiriz en espagnol) n'est qu'à une paire de kilomètres des redoutes de Trona et de Lindux. 


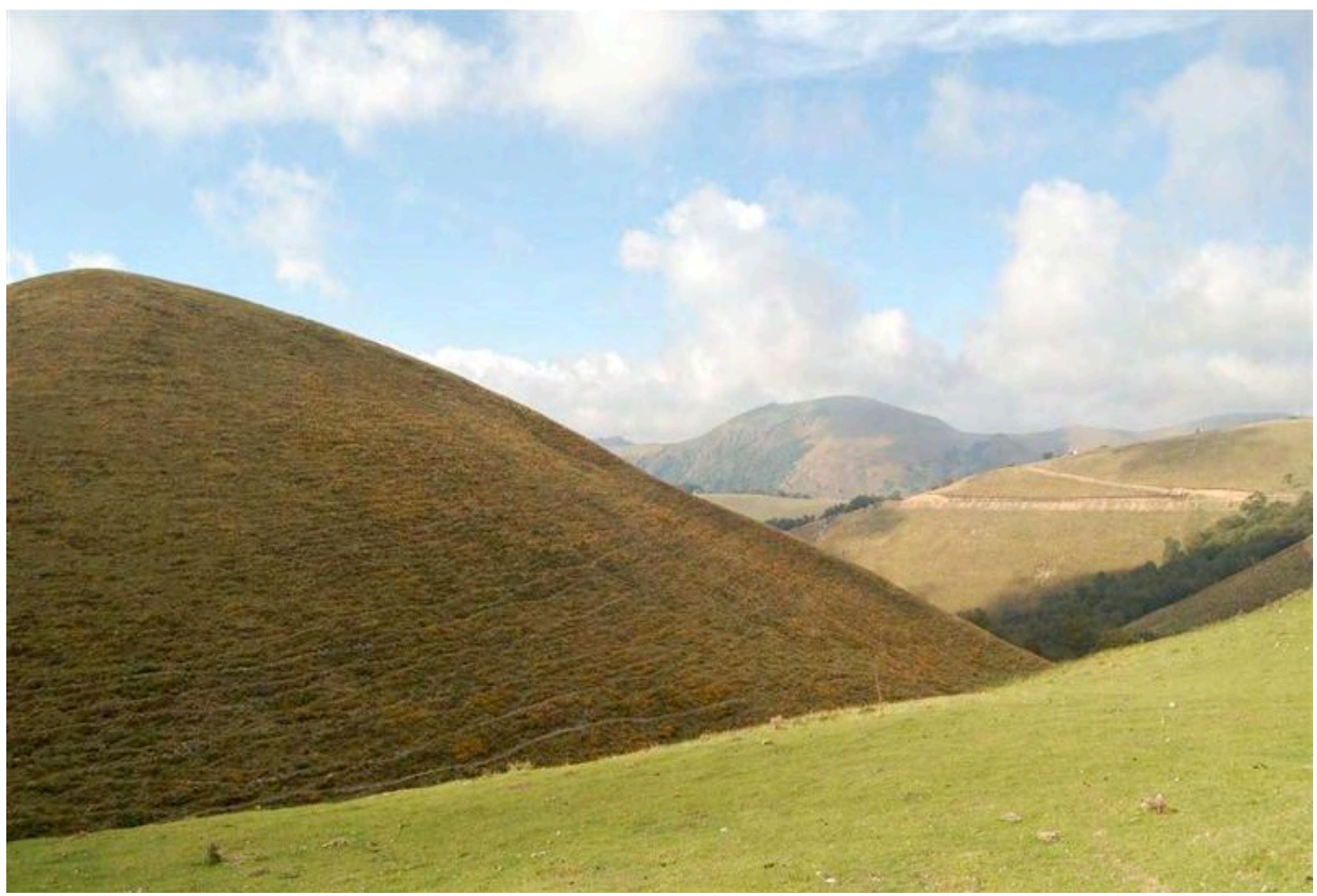

Figure 3. Prise de vue depuis la Crête de Pellusegagna. Au troisième plan la crête d'Orgambidesca découpée par les pistes, l'une des stations les plus riches en Bombus jonellus, on distingue plus bas les prolongements de la forêt d'Iraty dans les vallons épargnés par les vents. (Photo David Genoud)

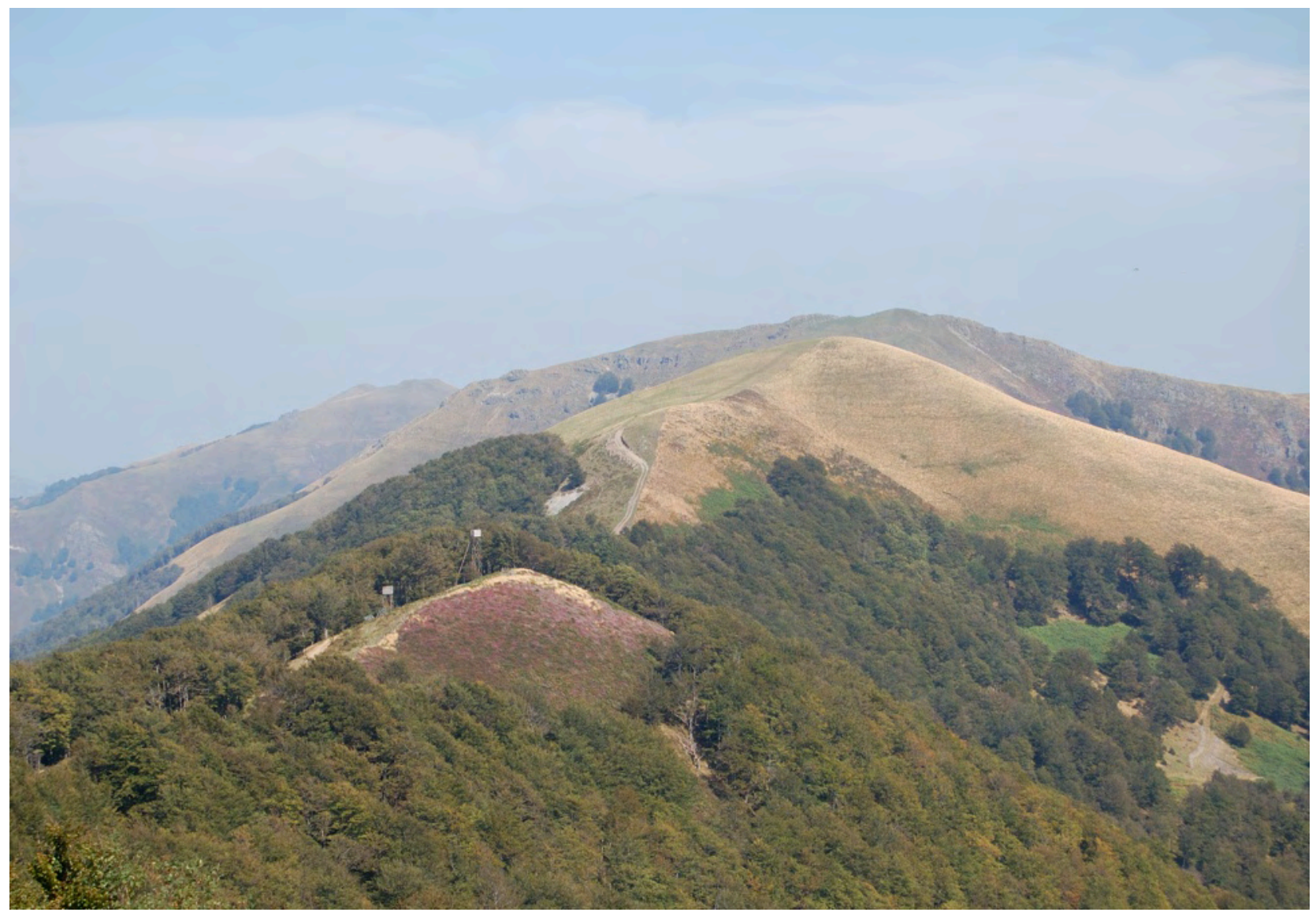

Figue 4. Prise de vue depuis la redoute de Trona. La crête d'Achistoy avec son flanc droit espagnol et son flanc gauche français. Achistoy le premier sommet pelé est abondamment pâturé côté Français. (Photo David Genoud) 


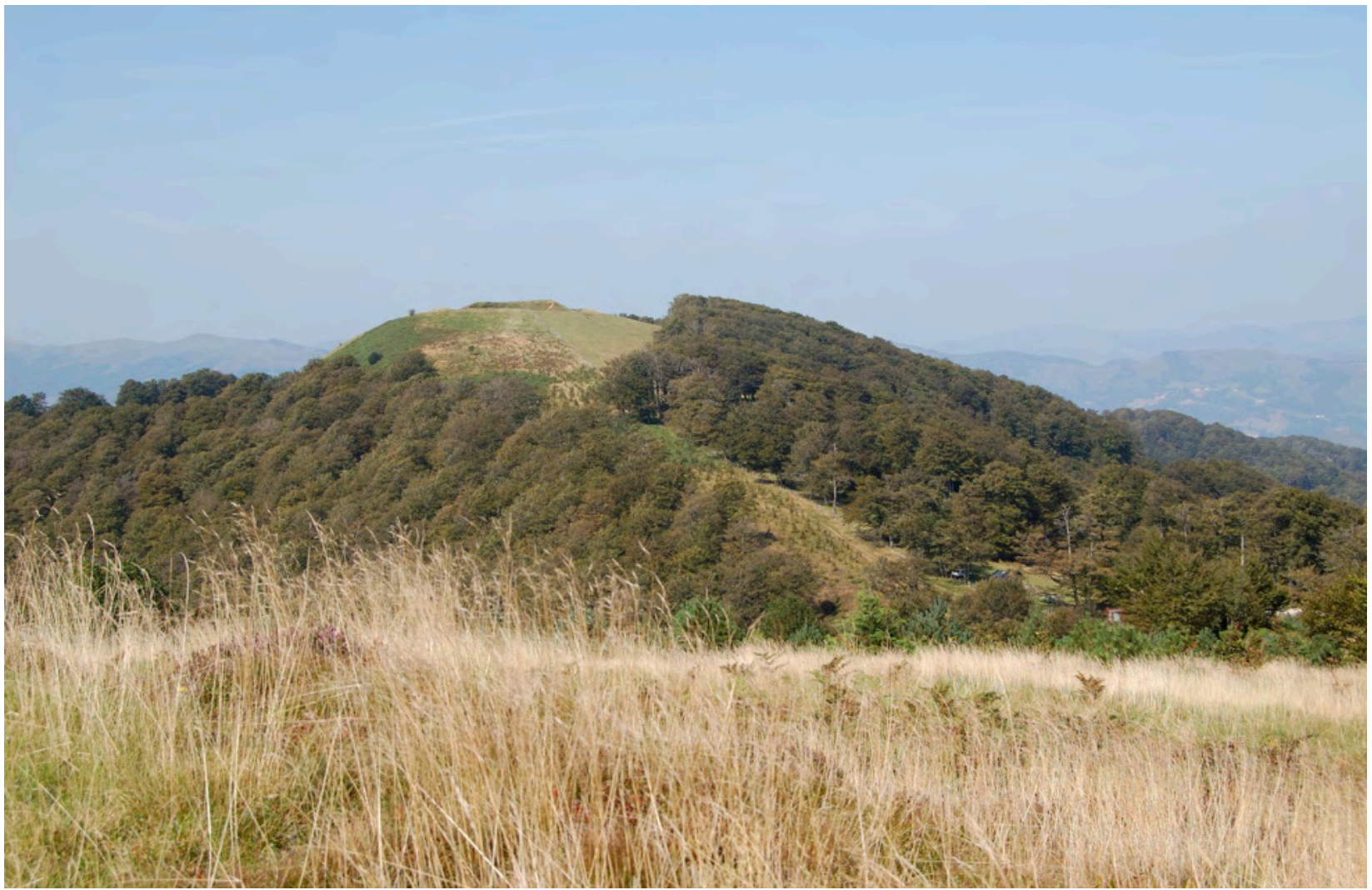

Figure 5. Prise de vue depuis la redoute de Trona. Vue sur la redoute de Lindux. On distingue là aussi nettement la limite frontalière avec l'effet pâturage. On observe aux premier et second plans les enrésinements de Pin sylvestre (Pinus sylvestris) sur le versant espagnol. (Photo David Genoud).

Il est intéressant de noter les disparités de localisation et d'abondance de l'espèce. Ainsi la forêt d'Hayra pourtant très favorable n'accueille pas l'espèce, mais son altitude (environ 800 mètres) exclut la présence de Vaccinum spp. Il en est de même des Col de Burdincurutcheta (Mendive) et de la tourbière de Surzay, qui présente des landes fractionnées et en coussinets. Les mâles isolés, trouvés sur ces stations, étaient toujours dans les zones de plus fortes densités à Erica cinerea.

Les femelles ont été contactées uniquement sur la redoute de Trona, la crête d'Achistoy, le col d'Oraaté, la crête d'Orgambidesca à Pellusegagna en présence d'abondantes stations à Calluna vulgaris qu'elles fréquentaient principalement.

Enfin la crête de Millagate et de Tharta composée de pâturage à Festuca sp. et Nardus stricta et de landes en coussinets d'Erica cinerea n'était pas fréquentée par Bombus jonellus. Cette crête est également bien plus isolée et lointaine des langues de boisements qui s'avancent des vallons abrités du vent vers les crêtes pâturées.

Cela laisse supposer que à l'exception de quelques mâles, Bombus jonellus ne s'éloignent guère des milieux les plus favorables (riches en ressources) a proximité des bois et lisières qu'il fréquente sans doute davantage au printemps et en début d'été puisque les Vaccinum spp. poussent sous couvert végétal, au minimum arbustif. Toutefois les investigations de juillet laissent indiquer que l'espèce ne descend pas non plus très bas dans la vallée du ruisseau de l'Iratiko où les ronciers abondent et où quelques landes richement fleuries d'Erica cinerea, Daboecia cantabrita et Calluna vulgaris ont été prospectées, sans succès, pour l'espèce. Toutefois nous émettrons une réserve à ces observations : et si fin juillet était une période de transition entre les 2 générations de Bombus jonellus?

On comprendra alors que ces nouvelles données restent encore bien trop fragmentaires pour appréhender la phénologie et en partie la biologie de l'espèce dans le massif Pyrénéen, biologie si particulière qui a caché ce bourdon aux regards des entomologistes pendant de nombreuses décennies.

En vallée des Aldudes, Bombus jonellus est nettement plus abondant sur les versants espagnols, pas que ces versants soient forcément exposés au Sud mais bien que l'activité agro-pastorale y est différente. Ainsi le pâturage apparaît comme très intensif sur tous les versants français, laissant des pelouses rases souvent érodées et vierges d'éricacées, sauf en de rares lisières. Le contraste est saisissant à Achistoy ou Lindux (voir Figure 4). Une certaine déprise agricole est décelable sur les versants espagnols. Déprise qui s'accompagne d'une politique d'aide financière à l'enrésinement par du Pin sylvestre (Pinus sylvestris) des pelouses seminaturelles sub-montagnardes. Si actuellement les versants espagnols sont plus favorables à Bombus jonellus, à terme, cet enrésinement pourrait lui être particulièrement néfaste (voir Figure 5). L'un de nous (PR) a observé ce phénomène dans la Margeride (Massif Central). Bombus jonellus s'y trouve seulement dans les terrains ouverts, à proximité de forêts. 


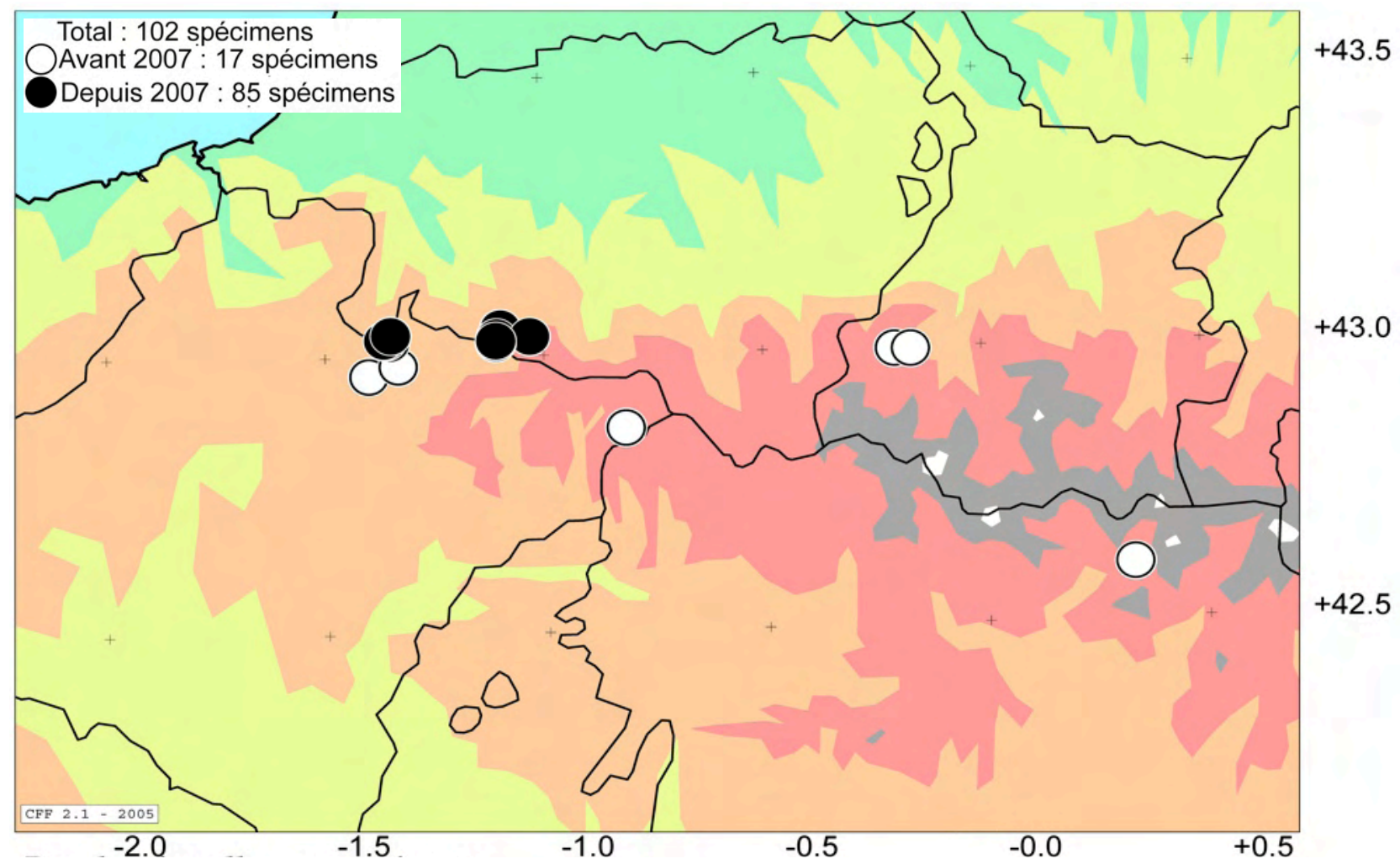

Figure 6. Distribution de Bombus jonellus dans les Pyrénées Occidentales. L'espèce n'est pas connue de la partie orientale (équidistances de 500 m).

Les zones récemment reboisées voient l'espèce disparaître, malgré la présence des Vaccinium spp. en sous-bois. En forêt d'Iraty, la situation est quelque peu identique d'un point vue de la pression pastorale. On note un surpâturage érodant le sol, parfois même en hêtraie, réduisant les surfaces en Myrtilles (Vaccinum myrtillus) et offrant de vastes pâturages herbacés pauvres en plantes à fleurs (éricacées, fabacées, apiacées, etc.). C'est à la lisière des forêt que l'on trouve des landes, souvent très rases à éricacées et ajoncs de Le Gall (Ulex gallii), c'est aussi là que se concentre la population automnale de Bombus jonellus. On peut supposer qu'une charge pastorale moins lourde favoriserait la population de Bombus jonellus sur ce massif, au moins transitoirement car si les sites se reboisent la lande disparaît. En raison de la rareté de l'espèces dans les Pyrénées, il est difficile de faire des recommendations plus précises pour les gestionnaires d'espaces naturels. Toutefois, il est clair que le reboisement des milieux ouverts est nuisible à Bombus jonellus en général. Cette pratique est donc à éviter.

\section{Références bibliographiques}

Alford DV, 1975. Bumblebees. Davis-Poynter, London, 352 pp. Amiet F 1996. Hymenoptera Apidae, 1. Teil. Allgemeiner Teil, Gattungsschlüssel, die Gattungen Apis, Bombus und Psithyrus. Insecta Helvetica 12, Neuchâtel, 98 pp.

Delmas R, 1976. Contribution à l'étude de la faune française des Bombidae (Hymenoptera, Apoidea, Bombidae). Annales de la Société entomologique de France (N.S.) 12: 247-290.

Gosselin M, Iserbyt S \& Rasmont $\mathbf{P}, 2007$. Faunistique des bourdons (Hymenoptera, Apoidea) de la vallée de Nohèdes (France, Pyrénées-Orientales) et des zones limitrophes. Notes Fauniques de Gembloux 60(1):13-23.

Iserbyt S, Durieux E-A \& Rasmont P, 2008. The remarkable diversity of bumblebees (Hymenoptera: Apidae: Bombus) in the
Eyne Valley (France, Pyrénées-Orientales). Annales de la Société entomologique de France (n.s.) 44 (2) : 211-241.

Iserbyt S, sous presse. La faune des bourdons (Hymenoptera: Apidae) du Parc National des Pyrénées occidentales et des zones adjacentes. Annales de la Société entomologique de France (N.S.), 58 dact. p.

Mahé G. \& Rasmont P, 2008. Apoidea Armoricana. http:// www.zoologie.umh.ac.be/hymenoptera/page.asp?id=112 (accès 20 février 2009).

Løken A, 1973. Studies on Scandinavian Bumble Bees (Hymenoptera, Apidae). Norsk entomologisk Tidsskrift 20(1): $1-218$.

Ornosa Gallego C, 1984. La subfamilia Bombinae (Hym., Apidae) de la fauna española. Thèse, Universidad Complutense de Madrid, $333 \mathrm{pp}$.

Pérez J, 1890. Catalogue des mellifères du Sud-Ouest. Actes de la Société Linnéenne de Bordeaux 44 : 133-200

Pérez J, 1905. Supplément au catalogue des mellifères du Sud-

Ouest. Actes de la Société Linnéenne de Bordeaux 59 : 5-7.

Rasmont P, 1986. Pyrobombus jonellus (KIRBY) (Hymenoptera: Apidae, Bombinae) dans la péninsule ibérique et les Pyrénées. Entomologische Berichte, Amsterdam 46(12): 185-189.

Rasmont P, 1988. Monographie écologique et biogéographique des bourdons de France et de Belgique (Hymenoptera, Apidae, Bombinae). Thèse de doctorat, faculté des Sciences Agronomiques de l'Etat, Gembloux, 309 + LXII p.

Rasmont P, 1999. Rapport préliminaires sur la faune des bourdons (Hymenoptera, Bombinae) des Pyrénées-Orientales; réserves de la Massane et du Vallon d'Eyne. Travaux de la Réserve naturelle de la Massane, Banyuls-sur-Mer 52: 1-17, 2 pls

Rasmont P, Delmas R \& Iserbyt S, 2002. Bombus (Pyrobombus) jonellus, carte de distribution. http:// www.zoologie.umh.ac.be/hymenoptera/photo.asp? ID = 255 (Atlas Hymenoptera, accès 20 février 2009).

Reinig WF 1976. Über die Hummeln und Schmarotzerhummeln von Nordrhein-Westfalen (Hymenoptera, Bombidae). Bonner zoologische Beiträge 27: 267-299.

Williams PH, 1998. An annotated checklist of bumble bees with an analysis of patterns of description (Hymenoptera: Apidae, Bombini). Bulletin of The Natural History Museum, Entomology Series 67(1): 79-152.

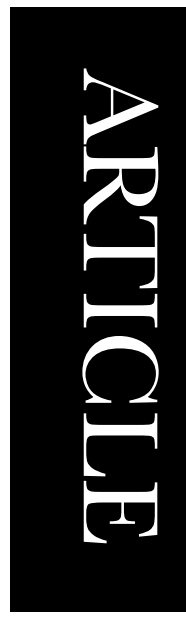

